Full length article

\title{
Generation of frequency tunable femtosecond pulses in a cw pumped linear dispersion-balanced passive mode-locked rhodamine $6 \mathrm{G}$ dye laser
}

\author{
M. Wittmann. A. Penzkofer and W. Bäumler \\ Naturwissenschaftliche Fakultät II-Phı̣sik. Universität Regensburg, W-8400 Regensburg, (ierman!.
}

Received 28 October 1991: revised manuscript received 24 January 1992

\begin{abstract}
Frequency tunable femtosecond light pulses are generated in a cw pumped linear group velocity dispersion balanced passive mode-locked rhodamine $6 G$ dye laser by birefringent filter tuning. The saturable absorhers $D O D C I$ (duration $\triangle t_{1}<100$ is in frequency region between $607 \mathrm{~nm}$ and $620 \mathrm{~nm}), \mathrm{DQOCI}\left(\Delta t_{\mathrm{L}}<100\right.$ fs between $585 \mathrm{~nm}$ and $\left.h 15 \mathrm{~nm}\right)$. and TCVEBI $\left(\Delta t_{1}<11\right)(1)$ is between $585 \mathrm{~nm}$ and $615 \mathrm{~nm}$ ) in ethỵlene glycol were applied.
\end{abstract}

\section{Introduction}

In passively mode-locked and hybridly modelocked dye lasers. frequency tunable subpicosecond pulses in the wavelength region between $488 \mathrm{~nm}$ and $905 \mathrm{~nm}$ (with gaps) have been generated by application of various amplifying-dye-saturable-absorber combinations and frequency tuning techniques (data are collected in reviews $[1-3]$, for recent papers see refs. [4-8]). The generation of frequency tunable pulses below $100 \mathrm{fs}$ in $\mathrm{cw}$ pumped passively modelocked $[9-12]$ and hybridly mode-locked dye lasers $[13]$ is reported scarcely. The obtained results are collected in table 1 . The laser frequency was varied by changing the saturable absorber concentration $[9.12]$ and it was tuned by dispersion adjustment [9.10.12.13].

In this paper a cw pumped linear dispersion-balanced passive mode-locked rhodamine $6 \mathrm{G}$ dye laser (abbreviation: DPM laser or DBPM laser) is used with the saturable absorber near the colliding-pulse mode-locking (CPM) position [12]. The group velocity dispersion balancing is achieved by proper adjusting the laser path through the prism-pair arrangement in the laser (prism-pair balanced operation). The saturable absorbers DODCI (3.3'-diethyloxadicarbocyanine iodide) [14], DQOCI (diethylquinoloxacarbocyanine iodide ) [15]. and TCVEBI
(2-[2-(2,3,6.7-tetra-hydro-1H.5H-benzo[i,j]chinolizin-9-yl)vinyl ]-3-ethylbenzothyazolium iodide. from Delta Agency. Sofia ) [5] dissolved in ethylene glycol are applied. The frequency tuning is achieved by inserting a birefringent filter in the optical path between the absorber cavity and the prism-pair arrangement. The tuning region is influenced by the saturable absorber concentration in the loss jet. The birefringent filter tuning results are compared with frequency tuning results obtained by aperturing the laser beam between the prism-pair and the back-reflecting mirror.

\section{Experimental facts}

\subsection{Experimental arrangement}

The experimental setup is shown in fig. 1. The femtosecond laser consists of a linear six-mirror resonator with the gain cavity in the quarter resonator length position, the loss cavity in the resonator center position (absorber jet approximately at CPM position), the birefringent filter mounted in a rotation stage under Brewster angle. and the prism-pair adjusted to the group velocity dispersion balanced position (generation of shortest stable soliton-like pulses [12]). The gain jet thickness is $d_{\mathrm{g}} \approx 250 \mu \mathrm{m}$ and the 
Table I

Frequency tunable sub- 100 fs dye lasers.

\begin{tabular}{|c|c|c|c|c|c|}
\hline Dye & Absorber & Tunning range "1 [nm] & Resonator & Tuning element & Ref. \\
\hline \multicolumn{6}{|c|}{ Passively mode-locked } \\
\hline Coumarin 102 & $\mathrm{DOCI}$ & $493-502$ & ring DB-CPM & $\begin{array}{l}\text { aperture in prism } \\
\text { quadrupole }\end{array}$ & {$[10]$} \\
\hline \multirow{6}{*}{ Rhodamine $6 \mathrm{G}$} & DODCI & $615-620$ & ring DB-CPM & prism shift and abs. conc. & [9] \\
\hline & DODCI & $614-620$ & linear DBPM & absorber conc." & {$[12]$} \\
\hline & TCETI ${ }^{\text {b) }}$ & $630-640$ & ring DB-CPM & absorber conc. ${ }^{\prime}$ & [11] \\
\hline & DODCI & $607-620$ & linear DBPM & BRF and abs.conc. & [this work] \\
\hline & DQOCI & $585-615$ & linear DBPM & BRF and abs.conc. & [this work] \\
\hline & TCVEBI & $585-615$ & linear DBPM & BRF & [this work] \\
\hline \multicolumn{6}{|c|}{ Hihridly mode-locked } \\
\hline Styryla 9 & IR I 40 & $840-880$ & ring DB-CPM & dispersion adjustment & $\mid 13\}$ \\
\hline
\end{tabular}

DB-CPM: colliding pulse mode-locked laser group velocity dispersion balanced with prisms. DBPM: passive mode-locked laser group velocity balanced using a prism pair, with absorber jet approximately in middle of resonator. BRF: single-plate birefringent filter.

"Within this tuning range it is $\Delta t_{1} \leq 100$ fs. "Anion is tetrafluoroborate.

"Peak wavelength changes with saturable absorber concentration; for fixed concentration laser wavelength is fixed.

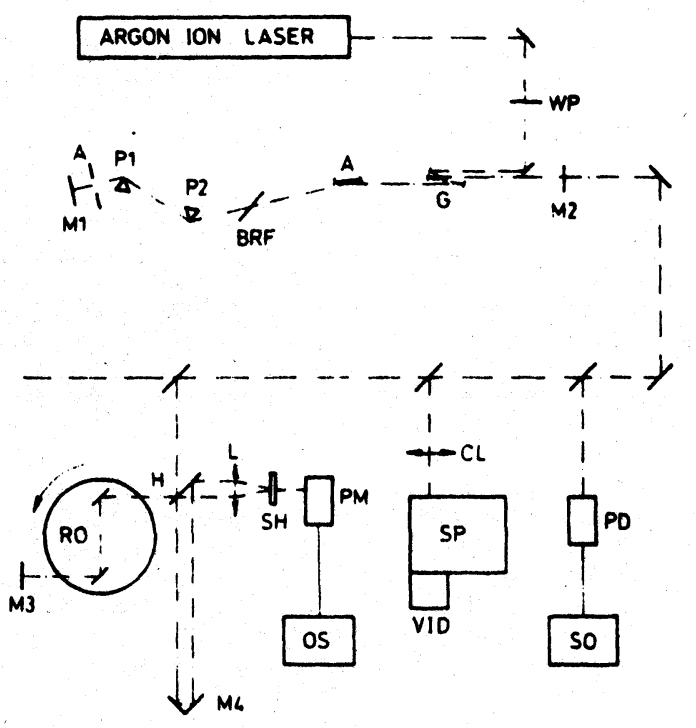

Fig. I. Experimental setup. WP. half-waveplate. M1. $100 \%$ mirror. M2, output mirror. G. gain cavity. A, absorber cavity. BRF. birefringent filter. P1. P2. Brewster prisms. A. spatial filter. PD. fast photodetector. SO, sampling oscilloscope. CL. cylindrical lens. SP. grating spectrometer. VID, vidicon. $H$. $50 \%$ beam splitter. RO, rotating mirror disc. M3. M4, 100\% mirrors. L. lens. SH, KDP-crystal $\left(\mathrm{KH}_{2} \mathrm{PO}\right.$ ). PM. photomultiplier (RCA IP28A). OS. digital oscilloscope (Gould Model DSO $400.100 \mathrm{MHz}$ bandwidth ). absorber jet thickness is $d_{\mathrm{a}} \approx 35 \mu \mathrm{m}$. For the prismpair dispersion balanced laser operation a detuning of the saturable absorber jet out of the CPM position has no influence on the laser periormance as was shown in ref. [12].

The continuous pulse trains are measured with a fast photodetector (Antel Model +R-S2, risetime 35 ps) and monitored on a sampling oscilloscope (Tektronix Scope 7603 with 7T11A sampling sweep unit. 7S11 sampling unit. and S-4 sampling head, rise time 25 ps).

The pulse durations are measured with a non-collinear background-free second harmonic rotatingmirror intensity autocorrelator [16]. The arrangement is sketched in fig. 1 . The relation between autocorrelation time $\Delta \tau_{\mathrm{L}}(\mathrm{fwhm})$ and pulse duration $\Delta t_{L}$ (fwhm) depends on the temporal pulse shape. It is given by $\gamma=\Delta \tau_{L} / \Delta t_{L}=2^{1 / 2}$ for gaussian pulses and $\gamma=1.543$ for $\operatorname{sech}^{2}$ pulses [1]. The relation for sech' pulses is used in this paper.

The pulse spectra are measured with a grating spectrometer and a vidicon detection system. The bandwidth product $\Delta \nu_{t} \Delta t_{t}$ of bandwidth-limited pulses depends slightly on the temporal pulse shape. It is $\Delta \nu_{L} \Delta l_{L}=0.441$ for gaussian pulses. and $\Delta \nu_{L} \Delta i_{1}=$ 0.315 for sech' pulses [1]. For time-chirped pulses (duration broadened by group velocity dispersion ) and for frequency-chirped pulses (spectrum broadened by self-phase modulation ) the bandwidth prod- 
uct is increased beyond the bandwidth limiting value.

\subsection{Gain medium}

The gain medium is rhodamine $6 \mathrm{G}$ in ethylene glycol. The dye concentration is $C_{\mathrm{g}}=1.7 \times 10^{-3} \mathrm{~mol} /$ $\mathrm{dm}^{3}$. The gain medium is excited by the multi-line emission of a cw argon ion laser (Spectra-Physics Model 2016, maximum multi-line pump power is 5 W). The absorption cross-section spectrum and the stimulated emission cross-section spectrum of rhodamine $6 \mathrm{G}$ in ethylene glycol are included in fig. 2 (taken from ref. [17]).

\subsection{Loss media}

The apparent absorption cross-section spectra of the applied saturable absorbers in ethylene glycol are shown in fig. 2. The DODCl absorption spectrum peaks at $\lambda=585 \mathrm{~nm}$ and belongs to the $\mathrm{N}$-isomer. The long-wavelength absorption shoulder around 630 $\mathrm{nm}$ is due to the absorption of thermally generated P-isomers [18]. The absorption peak of DQOCl is at $\lambda=600 \mathrm{~nm}$. and the absorption peak of TCVEBI is at $\lambda_{\mathrm{L}}=573 \mathrm{~nm}$.
The absorption recovery times of DODCI in ethylene glycol are $\tau_{A}(\mathrm{~N}$-isomer) $=1.3 \mathrm{~ns}$ (fluorescence quantum yield $\left.q_{\mathrm{N}}=0.55\right)$ and $\tau_{\mathrm{A}}(\mathrm{P}$-isomer $)=1.4 \mathrm{~ns}$ $\left(q_{\mathrm{P}}=0.35\right)$ [19]. For DQOCl in ethylene glycol and for TCVEBI in ethylene glycol we measured the fluorescence quantum efficiencies $q$ in this work using a self-assembled spectro-fluorimeter [20]. In the case of DQOCI we obtained $q \approx 0.04$ (excitation wavelength was $575 \mathrm{~nm}$ ) giving a fluorescence lifetime of $\tau_{\mathrm{F}}=q \tau_{\mathrm{rad}} \approx 160 \mathrm{ps}\left(\tau_{\mathrm{rad}} \approx 4 \mathrm{~ns}\right)$. The absorption recovery dynamics of $D Q O C I$ in various alcohols was studied in ref. [21]. For TCVEBI we obtained $q \approx 0.048$ (excitation wavelength $575 \mathrm{~nm}$ ) giving $\tau_{\mathrm{F}} \approx 180 \mathrm{ps}\left(\tau_{\mathrm{rad}} \approx 3.8 \mathrm{~ns}\right)$.

\subsection{Resonator mirrors}

The wavelength dependent reflectivity $p_{1}$ of our output mirror is displayed by the dashed curves in fig. 3a. and the total reflectivity of the six resonator mirrors. $\rho_{\mathrm{m}}=\Pi_{i=1}^{\mathrm{o}} \rho_{1}$, is shown by the solid curve in fig. 3a. The decreasing reflectivity at the long-wavelength and at the short-wavelength side sets an ultimate limit of the frequency tuning range.

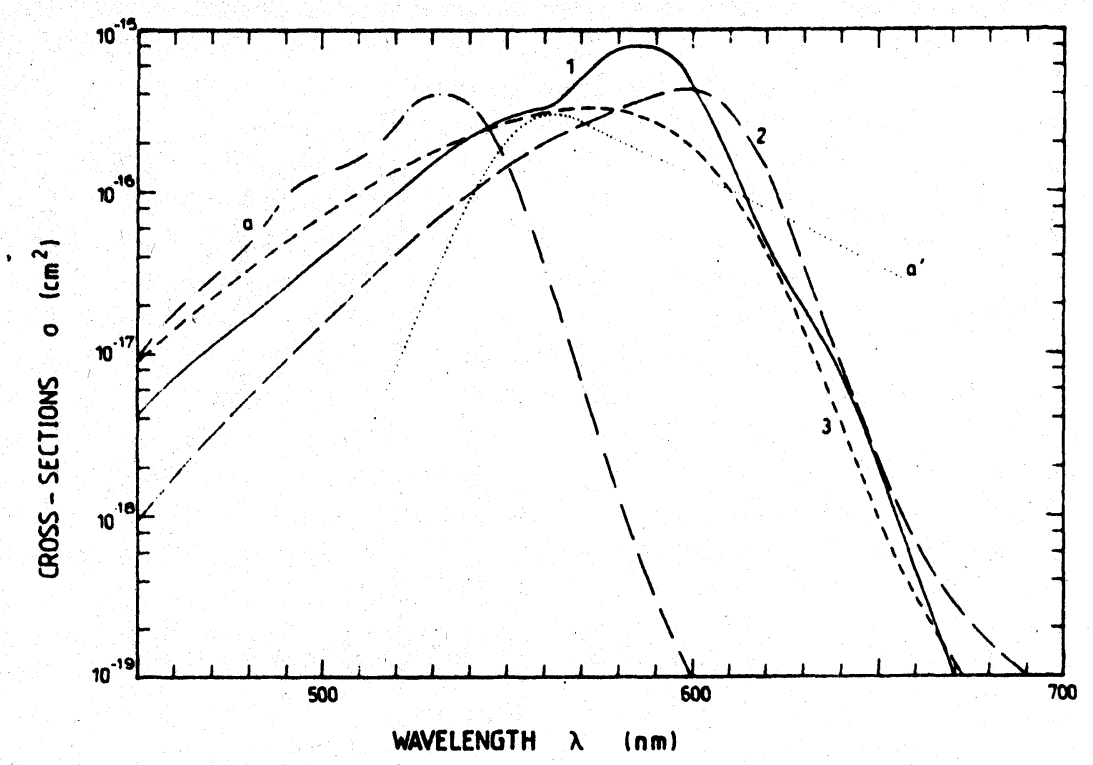

Fig. 2. Absorption cross-section spectra of rhodamine $6 G$ (a), DODCI (1), DQOCI (2), TCVEBI (3), and emission cross-section spectrum of rhodamine 6G ( $\left.a^{\prime}\right)$. Solvent is ethylene glycol. 


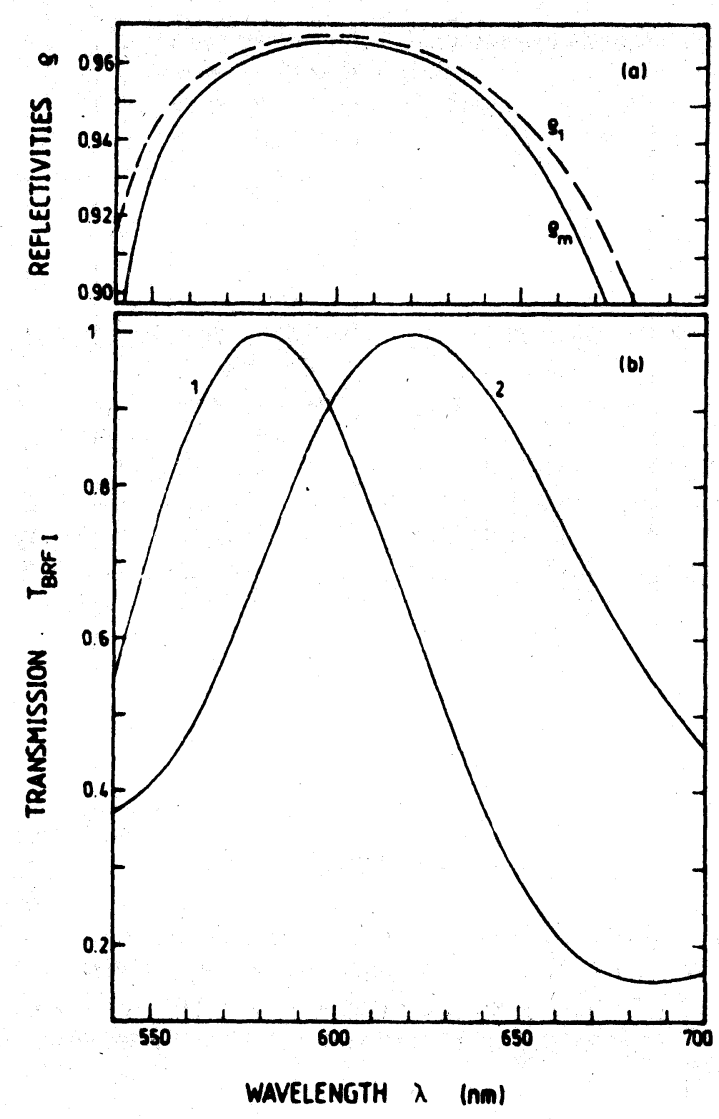

Fig. 3. (a) Reflectivity of output mirror, $p_{1}$. and total reflectivity, $\rho_{m}=\Pi_{i=1}^{i} p_{1}$. of all mirrors of the laser oscillator. (b) Calculated round-trip transmission caused by single-plate birefringent filter of thickness $d_{B R F}=126 \mu \mathrm{m}$. The azimuthal angles are $\phi=44.11^{\circ}$ (curve 1), and $\phi=51.27^{\circ}$. Refractive index data of quartz crystal are taken from ref. [42].

\subsection{Birefringent filters}

Two single plate quartz crystal birefringent filters of different thickness and optic axis orientation were applied. The plate BRF I had a thickness of $d_{B R F I}=126 \mu \mathrm{m}$ and the optic axis oa was parallel to the entrance and exit surfaces (optic axis at angle of $\Psi=90^{\circ}$ to surface normal). The plate BRF II had a thickness of $d_{\text {BRF II }}=2 \mathrm{~mm}$ a $\quad$ optic axis had an angle of $\Psi=90^{\circ}+\psi=150^{\circ}$ the surface normal.

Most experiments were carried out with BRF I. Its alignment in the resonator and the relevant angles are shown by the inset of fig. 4 . The theory of wavelength tuning with birefringent filters having their optic axis in the entrance surface $\left(\Psi=90^{\circ}, \psi=0\right)$ is given in ref. [22-24]. In fig. $3 b$ two wavelength dependent round-trip transmission curves $T_{\mathrm{BRF}}$ for the filter BRF $I$ in the femtosecond laser oscillator are shown. The filter is tuned to peak transmission at either $580 \mathrm{~nm}$ (curve 1) or $620 \mathrm{~nm}$ (curve 2). The total round-trip light retainment $R$ of the resonator is $R=\rho_{\mathrm{m}} T_{\mathrm{BRF}}$. The wavelength position of the peak value of $R(\lambda)$ together with the net spectral amplification profile of the gain and loss medium determine the lasing wavelength.

The tuning possibility of birefringent filters with $\psi=0^{\circ}$ as a function of thickness is illustrated in fig. 4. For the solid curves it is $T_{\mathrm{BRF}}(550 \mathrm{~nm})=1$. and for the dashed curves it is $T_{\mathrm{BRF}}(650 \mathrm{~nm})=1$. The hatched regions cover $T_{\mathrm{BRF}}=1$ over the wavelength region from $550 \mathrm{~nm}$ to $650 \mathrm{~nm}$. The interference order $\boldsymbol{m}$ gives the optical path-length difference between the extra-ordinary ray and the ordinary ray in units of the wavelength [22-24]. For $d_{\text {BRF }} \leqslant 50 \mu \mathrm{m}$ and for $86 \mu \mathrm{m} \leqslant d_{\mathrm{BRF}} \leqslant 100 \mu \mathrm{m}$ no birefringent filter tuning in the wavelength region between $550 \mathrm{~nm}$ and $650 \mathrm{~nm}$ is possible (there exists no azimuthal angle $\phi$ where $T_{\mathrm{BRF}}=1$ ).

The thin birefringent filter BRF I has a small etalon effect (see inset of fig. $9 \mathrm{~b}$ ) which modulates the pulse spectra and causes weak delayed pulses. The frequency spacing of the modulated spectra is

$\delta \tilde{\nu}=\frac{1}{\delta l_{\mathrm{opt}}}=\frac{1}{2 d_{\mathrm{BRF}}\left(n^{2}-\sin ^{2} \theta_{1}\right)^{1 / 2}}$,

where $\delta l_{\text {opt }}$ is the optical length for a double passage through the filter, $n$ is the refractive index of the filter. and $\theta_{\mathrm{i}}=\arctan (n)=90^{\circ}-\theta^{\prime}$ is the Brewster angle of incidence $\left(n \approx 1.544, \delta \dot{\nu} \approx 30 \mathrm{~cm}^{-1}\right.$ for BRF 1$)$. The lateral displacement of the double reflected beam is

$h=\frac{2 d_{\mathrm{BRF}} \cos \theta_{\mathrm{i}}}{n}$

for Brewster angle incidence. For BRF I it is $h \approx 0.088$. $\mathrm{mm}$. The laser beam diameter at the birefringent filter position is $d_{L} \approx 0.5 \mathrm{~mm}$ [12]. The temporal delay of the double reflected light is

$\delta t=\delta l_{\text {opt }} / c_{0}=1 / c_{0} \delta \tilde{\nu}$.

which gives $\delta t \approx 1.1$ ps for BRF 1 .

For coherent gaussian pulses $I(t)=I_{0 \mathrm{~L}} \exp \left[-t^{2}\right)$ $\left.\left.t_{0}^{2}\right)\right]+I_{0 s} \exp \left[-(t-\delta t)^{2} / t_{0}^{2}\right]$, where $I_{0 L}$ is the peak 


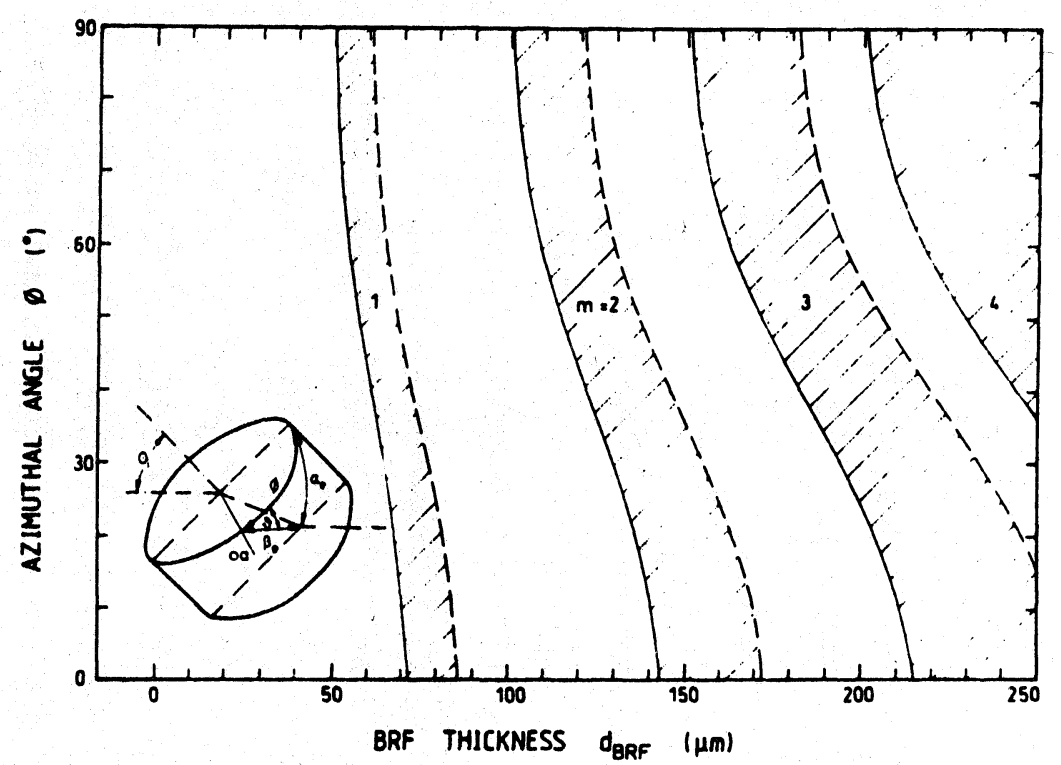

Fig. 4. Illustration of frequency tuning region of single plate birefringent filters as a function of plate thickness. Solid curves belong to $\lambda=550 \mathrm{~nm}$ and dashed curves belong to $i=650 \mathrm{~nm}$. The hatched regions cover the wavelength range between $550 \mathrm{~nm}$ and $650 \mathrm{~nm}$. $m$ is the interference order (see main text). The inset shows ray tracing through BRF with $\psi=0$ (optic axis oa in surface plane).

intensity of the main pulse and $I_{0 s}$ is the peak intensity of the satellite pulse, the modulated spectra were calculated by fast Fourier transformation. The spectral modulation depth $\kappa_{\nu}=\left[I_{\text {env.max }}(0)\right.$ $\left.-I_{\text {env.min }}(0)\right] / I_{\text {env.max }}(0)$ versus $I_{0 S} / I_{0 \mathrm{~L}}$ is shown in fig. 5 for $\Delta t_{L}=2[\ln (2)]^{1 / 2} t_{0}=70$ fs and $\delta t=1.1$ ps. $I_{\text {env.max }}$ and $I_{\text {env.min }}$ are the maximum and minimum envelope curves of the modulated spectral intensity $I(\nu)$. The lower inset in fig. 5 shows $I(t) / I(0)$ for a satellite pulse height of $I_{0 \mathrm{~s}} / I_{0 \mathrm{~L}}=0.05$, and the upper inset of fig. 5 shows the corresponding normalized spectrum $I(\nu) / I(0)$.

The single path etalon effect (generation of satellite pulses by double reflections) is negligible because the birefringent filter plate is oriented under Brewster angle. The measurable effect builds up due to the multiple passages in the resonator (satellite pulse enhancement by repetitive reflection accumulation similar to the sensitivity enhancement by intracavity absorption in lasers [25]).

The small satellite pulse formation by thin birefringent filters may be avoided by using birefringent filters of low interference order (large free spectral range ) but of a thickness $d_{\text {BRF }}$ larger than the laser beam diameter $d_{\mathrm{L}}$. These conditions are achieved by using a quartz plate with its optic axis being oblique to the plate entrance and exit surfaces (angle $\psi$ near to Brewster angle, see inset of fig. 7) [8.26-29].

For a birefringent filter with the optic axis oa at an angle of $\Psi=90^{\circ}+\psi$ to the surface normal the theoretical treatment of ref. [24] remains valid, if $\beta_{\mathrm{e}}=\beta_{\mathrm{e}}(\psi=0)$ with $\cos \beta_{\mathrm{e}}=\cos \phi \cos \alpha_{\mathrm{e}}$ is replaced by $\beta_{\mathrm{e}}(\psi)=\beta_{\mathrm{e} . \psi}$ with

$\cos \beta_{\mathrm{e} . \psi}=\cos \beta_{\mathrm{e}} \cos \psi+\sin \beta_{\mathrm{e}} \sin \psi \cos \%$

$$
=\cos \beta_{\mathrm{e}} \cos \psi+\sin \psi \sin \alpha_{\mathrm{e}}
$$

(spherical trigonometry with $\gamma=90^{\circ}-\vartheta$ and $\cos ;$ $=\sin \theta=\sin \alpha_{e} / \sin \beta_{e}$, where $\theta$ is the angle between $\beta_{\mathrm{e}}$ and $\left.\phi\right)$. The angles are defined in the insets of fig. 4 and fig. 7.

In fig. 6 the ratios of the phase differences

$r(\psi, \phi)=\frac{\delta_{e}(\psi, \phi)-\delta_{0}}{\delta_{e}\left(0.90^{\circ}\right)-\delta_{0}}=\frac{m(\psi, \phi)}{m\left(0,90^{\circ}\right)}$

(eq. (4) of ref. [24]) versus angle $\psi$ are displayed for various azimuthal angles $\phi$. The ratio of the phase differences $r$ gives the ratio of the interference orders $m(\psi, \phi) / m\left(0,90^{\circ}\right)$. In order to obtain the same interference order (birefringent filter effect) for an op- 


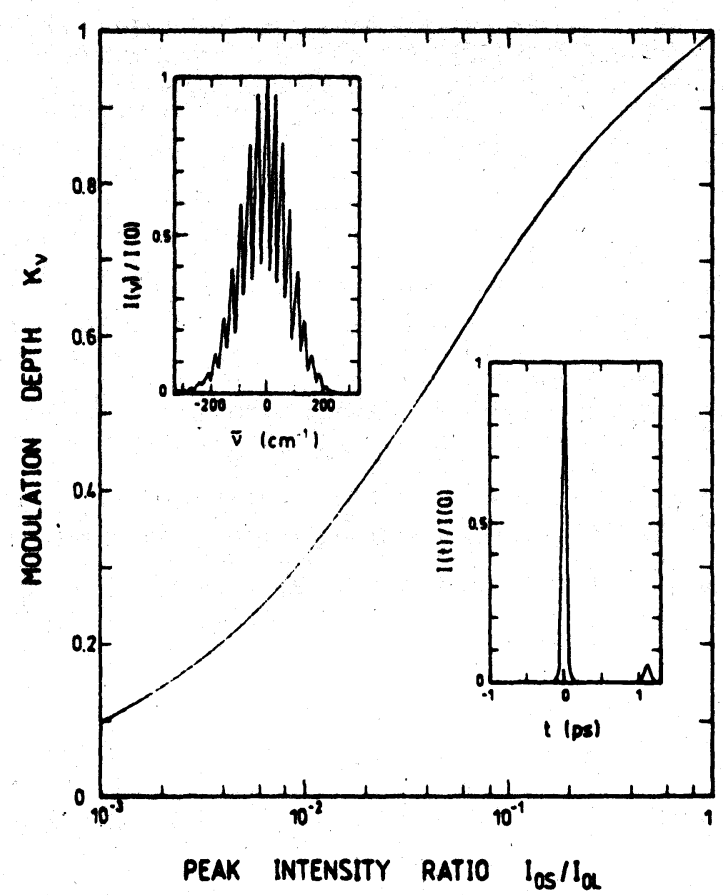

Fig. 5. Spectrum modulation depth, $\kappa_{v}$, versus peak intensity ratio of satellite pulse to main pulse, $I_{0 S} / I_{01}$. Temporal gaussian pulses of $\Delta l_{L}=70 \mathrm{fs}$ ( $\mathrm{fwhm}$ ) and of $\delta t=1.1$ ps separation are used in calculations. The insets belong to $I_{0 S} / I_{0 L}=0.05$.

tic axis angle orientation of $\psi=\psi_{11}$ and $\phi=\phi_{11}$ as for $\psi=0$ and $\phi=\phi_{1}$, the plate thickness has to be choosen to be

$d_{\mathrm{BRF}}\left(\psi_{11}, \phi_{11}\right)=d_{\mathrm{BRF}}\left(0, \phi_{1}\right) \frac{r\left(0, \phi_{1}\right)}{r\left(\psi_{11}, \phi_{11}\right)}$

For our birefringent filter BRF II $(d=2 \mathrm{~mm}$, $\psi=60^{\circ}$ ) the lateral displacement (eq. $\left.(2)\right)$ is $h \approx 1.4$ mm. No temporal satellites and no spectral modulation occur with this filter (see below). Some roundtrip transmission curves $T_{\mathrm{BRF}} \mathrm{II}$ are shown in fig. 7 . The peak transmissions are set to $580 \mathrm{~nm}$ and 620 $\mathrm{nm}$. The curves apply to the interference orders $m=1$, 2 , and 3.

\section{Femtosecond laser tuning results}

In the frequency tuning studies of the femtosecond rhodamine $6 \mathrm{G}$ dye laser, three different saturable

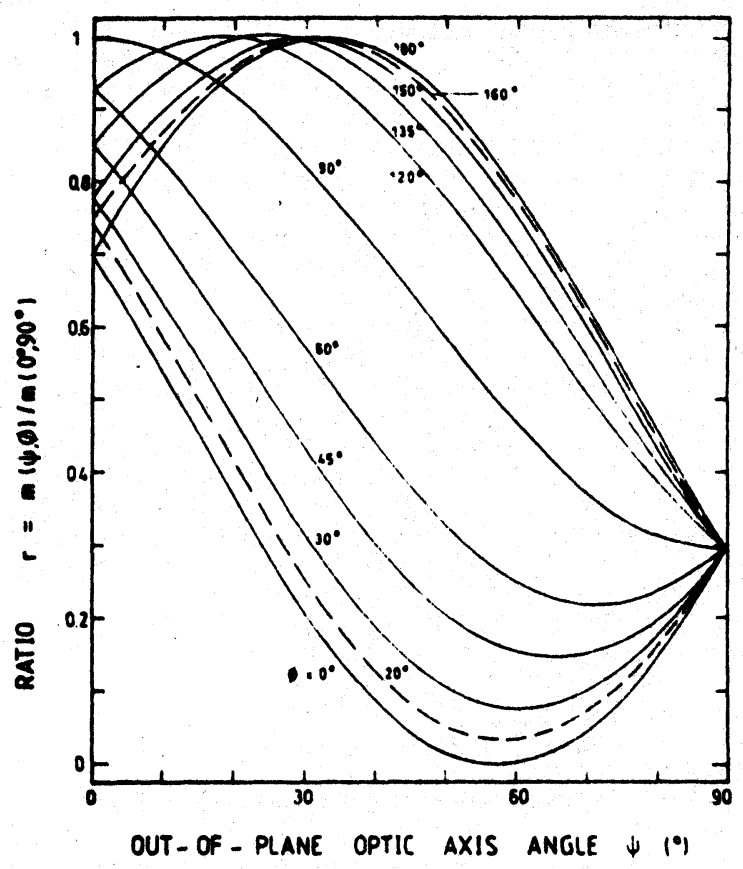

Fig. 6. Reduction of interference order $m$ for out-of-plane orientation of optic axis. The curves belong to different azimuthal angles $\phi$. Refractive index data of quariz crystal at $i=620 \mathrm{~nm}$ are used [42].

absorbers, DODCI, DQOCI. and TCVEBI. were applied. The saturable absorber concentration was varied to change the tuning range. The birefringent filter BRF I was used for all three saturable dyes. and the filter BRF II was applied for the absorber TCVEBI. Indications of the influence of positive self-phase modulation on the prism-pair balanced (soliton-like) pulse formation are given. The birefringent filter tuning was compared with the spatial filter tuning in the frequency dispersion region of the prism pair (aperture A between prism PI and mirror MI. see fig. 1). The saturable absorber DQOCI was used in the spatial filter tuning experiment.

\subsection{Saturable absorber DODCI}

In fig. 8 the wavelength tuning behaviour of the dispersion-balanced passive mode-locked (DBPM) rhodamine 6G laser with DODCI in ethylene glycol as saturable absorber is presented. The filter BRF I is angle tuned $(\phi)$ for the wavelength variation. The numbered curves belong to different saturable $a b$ - 


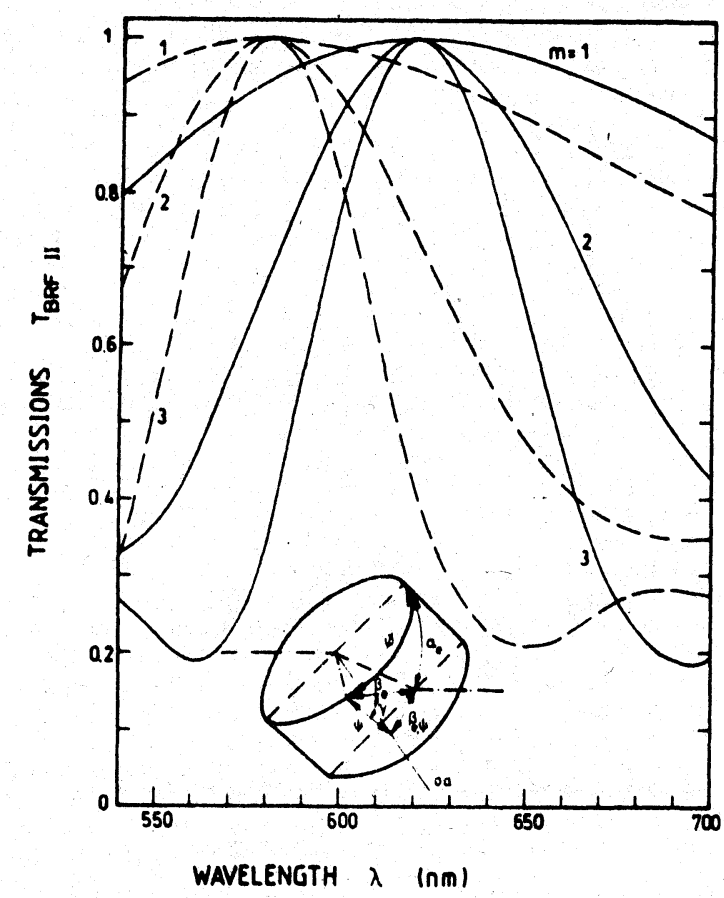

Fig. 7. Calculated round-trip transmission caused by single-plate birefringent filter BRF $\|$ of thickness $d_{\mathrm{BRF} \|}=2 \mathrm{~mm}$ and orientation of the optic axis of $\psi=60^{\circ}$. Solid curves. maximum transmission at $620 \mathrm{~nm}$ with azimuthal angles $\phi=18.01^{\circ}(m=1)$. $26.29^{\circ}(m=2)$. and $32.73^{\circ}(m=3)$. Dashed curves, maximum transmission at $580 \mathrm{~nm}$ with $\phi=17.32^{\circ}(m=1) .25 .29^{\circ}(m=2)$, and $31.49^{\circ}(m=3)$. The inset shows ray tracing through birefringent filter with $\psi \neq 0$.

sorber concentrations. The pulse duration $\Delta t_{\mathrm{L}}(\mathrm{a})$, the bandwidth product $\Delta \nu_{\mathrm{L}} \Delta t_{\mathrm{L}}$ (b), and the threshold pump power $P_{\text {pu.th }}(\mathrm{c})$ versus peak lasing wavelength $\lambda_{p}$ are displayed. The application of three saturable absorber concentrations extends the tuning range. For sub-100 fs pulses, the total tuning range is from $607 \mathrm{~nm}$ to $620 \mathrm{~nm}$.

The wavelength tuning under stable laser performance conditions is restricted to the long-wavelength absorption region of the $\mathrm{N}$-isomers of DODCI $\left(\lambda_{p}>600 \mathrm{~nm}\right)$. The photoisomerisation dynamics [18.19] seems to hinder a stable femtosecond pulse generation in the peak absorption region of the $\mathrm{N}$ isomer $(580 \mathrm{~nm}$ to $600 \mathrm{~nm})$. The absorption changes [19] and refractive index changes [17] by P-isomer accumulation seem to be responsible for this behaviour. At the long-wavelength side the tuning is lim-

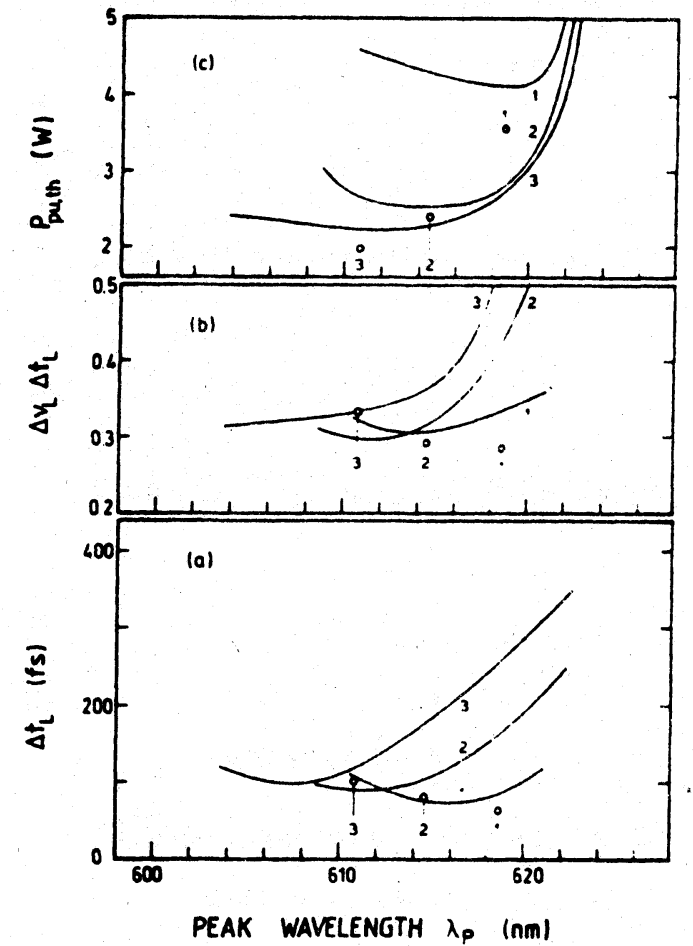

Fig. 8. Frequency tuning characteristics of saturable absorber DODCI and filter BRF I. The absorber concentrations are $C_{\mathrm{a}}=1.5 \times 10^{-4}(1), 9.1 \times 10^{-5}(2)$, and $5.1 \times 10^{-5}(3)$. The circles give the experimental results for the resonator without birefringent filter.

ited because the threshold pump power becomes higher than the available pump power.

The bandwidth product. $\Delta \nu_{\mathrm{L}} \Delta t_{\mathrm{t}}$. is smallest where the pulse durations are shortest. It increases towards the borders of high pump power.

The average femtosecond laser power outside the resonator is in the range between $20 \mathrm{~mW}$ and $5 \mathrm{~mW}$. It decreases with rising wavelength.

The laser data without birefringent filter are included by circles in fig. 8 . The pulse duration and the bandwidth product decrease with increasing absorber concentration. The soliton-like pulse formation in the prism-pair balanced group velocity dispersion operation regime $[30-32]$ is responsible for the short durations [12] and the small bandwidth product [33]. The saturable absorber action leads to asymmetric pulse shapes (steeper leading edge) which results in asymmetric spectra and smaller $\Delta \nu_{\mathrm{L}} \Delta t_{\mathrm{L}}$-products $[12,14,17,33,34]$. 
In fig. 9 examples of spectral shapes and auto-correlation traces are shown for a saturable absorber concentration of $C=1.5 \times 10^{-4} \mathrm{~mol} / \mathrm{dm}^{-3}$. Parts (a) show the results without birefringent filter while parts (b) show the results for the birefringent filter BRF I tuned to $617 \mathrm{~nm}$. The intra-cavity enhanced etalon effect of the thin birefringent filter BRF I shows up in the modulation of the spectral distribution with a modulation spacing of $\delta \tilde{\nu} \approx 30 \mathrm{~cm}^{-1}$. The modulation depth of the spectrum is $\kappa_{\nu} \approx 30 \%$ (upper part b of fig.9). The corresponding satellite pulse height is $I_{0 S} / I_{0 L} \approx 0.01$ according to fig.5. The signal height $S(\tau)$ of the background-free autocorrelation trace at the satellite pulse position relative to the signal height $S(0)$ at $\tau=0$ is [35] $S(\tau) / S(0) \approx I_{0 S} I_{0 L} /\left(I_{0 L}+I_{0 S}\right)^{2}$ $\approx I_{0 \mathrm{~s}} / I_{0 \mathrm{~L}}$. In the lower part of fig. $9 \mathrm{~b}$. the satellite pulse signal cannot be resolved from the noise level.

In all experiments with BRF I, $\kappa_{\nu}$ varied between
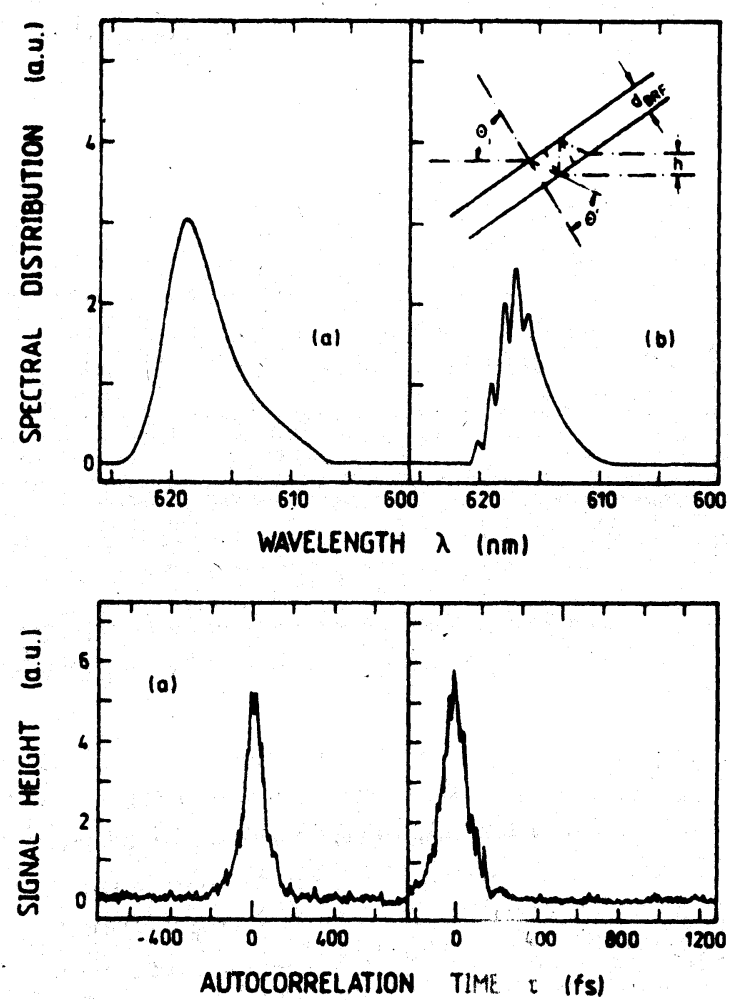

Fig. 9. Pulse spectra and autocorrelation traces for DODCl of concentration $C_{\mathrm{a}}=1.5 \times 10^{-4} \mathrm{~mol} / \mathrm{dm}^{3}$. (a) Without birefringent filter. (b) Birefringent filter BRF I tuned to peak laser wavelength $\lambda_{p}=617 \mathrm{~nm}\left(\phi=54^{\circ}\right)$. Ray tracing through birefringent filter is indicated by the inset.
0.25 and 0.7 and the observed satellite to main pulse ratio was in the range of $0 \leqslant I_{0 \mathrm{~S}} / I_{\mathrm{OL}} \leqslant 0.1$.

It is noted that the appearance of the satellite pulse has practically no influence on the temporal resolution in femtosecond time-resolved pump and probe experiments, since for events relaxing on a femtosecond time scale the event has disappeared before intervening with the satellite pulse. For events relaxing on a picosecond time scale a weak satellite pulse of one picosecond separation does not to influence measurably the pump-probe result.

\subsection{Saturable absorber $D Q O C I$}

The solid curves in fig. 10 show the pulse duration $\Delta t_{\mathrm{L}}$, the bandwidth product $\Delta \nu_{\mathrm{L}} \Delta t_{\mathrm{L}}$ and the pump power threshold $P_{\text {pu.th }}$ versus peak laser wavelength $\lambda_{p}$ for the filter BRF I. The DQOCI concentration is $C_{\mathrm{a}}=7.4 \times 10^{-5} \mathrm{~mol} / \mathrm{dm}^{3}$. The sub- 100 fs tuning range is from $591 \mathrm{~nm}$ to $614 \mathrm{~nm}$. Using $C_{\mathrm{a}}=$

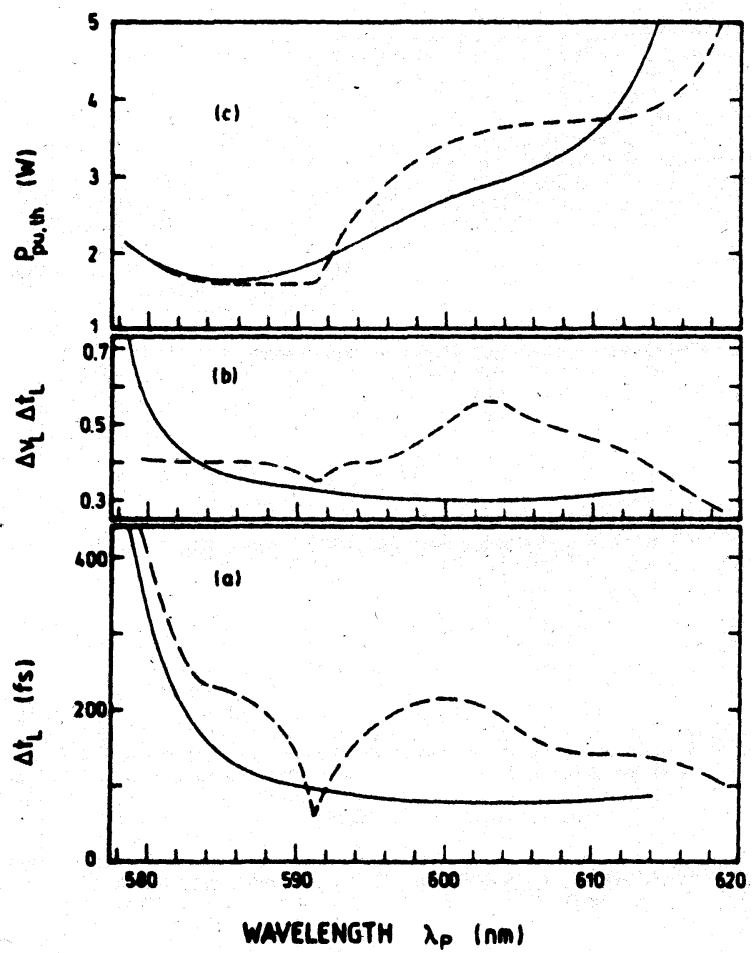

Fig. 10. Frequency tuning characteristics of saturable absorber DQOCI. Solid curves belong to BRF I and dashed curves to spatial filter tuning. The absorber concentration is $C_{2}=7.4 \times 10^{-s}$. 
$1.4 \times 10^{-4} \mathrm{~mol} / \mathrm{dm}^{3}$ a sub- 100 fs tuning range from $585 \mathrm{~nm}$ to $600 \mathrm{~nm}$ was obtained (curves not shown in fig. 10). The average power of the generated continuous femtosecond pulse trains was between $5 \mathrm{~mW}$ and $15 \mathrm{~mW}$.

Without birefringent filter the spectral intensity peak was at $\lambda_{\mathrm{p}}=590 \mathrm{~nm}$ for $C_{\mathrm{a}}=1.4 \times 10^{-4} \mathrm{~mol} / \mathrm{dm}^{3}$ and at $592 \mathrm{~nm}$ for $C_{\mathrm{a}}=7.4 \times 10^{-5} \mathrm{~mol} / \mathrm{dm}^{3}$. In both cases the measured pulse durations were $50 \mathrm{fs}$. The spectral halfwidth were $\Delta \tilde{\nu}_{\mathrm{L}}\left(1.4 \times 10^{-4} \mathrm{M}\right)=315$ $\mathrm{cm}^{-1}$ and $\Delta \tilde{\nu}\left(7.4 \times 10^{-4} \mathrm{M}\right)=265 \mathrm{~cm}^{-1}$ giving bandwidth products of $\Delta \nu_{\mathrm{L}} \Delta \mathrm{l}_{\mathrm{L}}\left(1.4 \times 10^{-4} \mathrm{M}\right)=0.47$ and $\Delta \nu_{\mathrm{L}} \Delta t_{\mathrm{L}}\left(7.4 \times 10^{-5} \mathrm{M}\right)=0.40$. The intra-cavity pulse durations are thought to be shorter than the measured durations because of the transit time broadening of the pulses by the positive group velocity dispersion of the output laser mirror and of the traversed optical glass of the autocorrelator [36]. tssuming inside the laser resonator $\Delta \nu_{\mathrm{L}} \Delta t_{\mathrm{L}}=0.315$, then intra-cavity pulse durations of $\Delta t_{\mathrm{L}}\left(1.4 \times 10^{-4}\right.$ $M)=33.5$ fs and $\Delta t_{L}\left(7.4 \times 10^{-5} M\right)=40$ fs are estimated. Around $585 \mathrm{~nm}$ the bandwidth of the net amplification profile of the gain medium and the mirror reflectivity seems to be broader than around $620 \mathrm{~nm}$. Therefore shorter pulses seem to be generated with DQOCI than with DODCI for our experimental parameters [12].

For $C_{\mathrm{a}}=7.4 \times 10^{-5} \mathrm{~mol} / \mathrm{dm}^{3}$ the laser stability without birefringent filter was not very high since there was some tendency to lase at $\lambda_{p} \approx 600 \mathrm{~nm}$ (accumulated spectra show peaks at $592 \mathrm{~nm}$ and 600 $\mathrm{nm}$ ).

\subsection{Saturable absorber TCVEBI}

Experiments with the thin filter BRF I and the thick filter BRF II were carried out. Indications of the influence of self-phase modulation on the prismpair balanced pulse generation are given.

In fig. 11. $\Delta t_{\mathrm{L}}, \Delta \nu_{\mathrm{L}} \Delta t_{\mathrm{L}}$, and $P_{\mathrm{pu}, \mathrm{th}}$ versus $i_{\mathrm{p}}$ are plotted for $C_{\mathrm{a}}=1.75 \times 10^{-4} \mathrm{~mol} / \mathrm{dm}$ (curves 1 ) and $C_{\mathrm{a}}=9 \times 10^{-5} \mathrm{~mol} / \mathrm{dm}^{3}$ (curves 2 ). The birefringent filter BRF I was used. The circles indicate the data obtained without birefringent filter. For the higher. absorber concentration pulse durations below $100 \mathrm{fs}$ were achieved over a wavelength region from $585 \mathrm{~nm}$ to $615 \mathrm{~nm}$

In fig. 12 the solid curves belong to the thick bi-

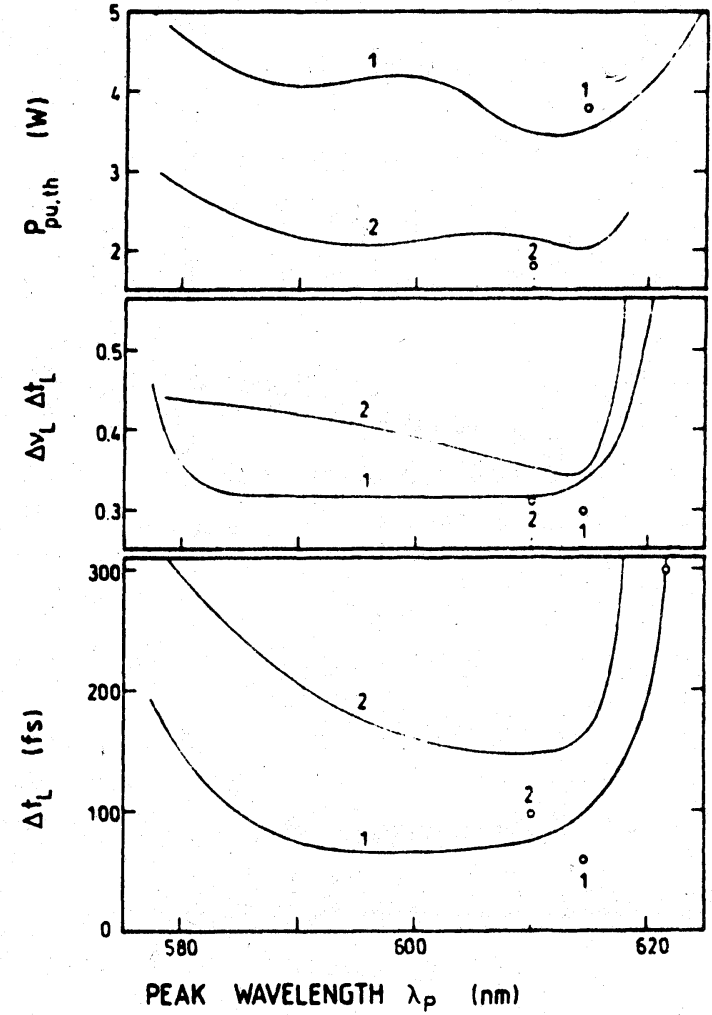

Fig. 11. Frequency tuning with saturable absorber TCVEBI. Curves belong to BRF I with absorber concentration $C_{2}=$ $1.75 \times 10^{-4} \mathrm{~mol} / \mathrm{dm}^{3}(1)$ and $C_{\mathrm{a}}=9 \times 10^{-5} \mathrm{~mol} \cdot \mathrm{dm}^{3}(2)$. Circles indicate the results without a birefringent filter.

refringent filter BRF II and the dashed curves to the thin birefringent filter BRF I. The saturable absorber concentration was set to $C_{\mathrm{a}}=9 \times 10^{-5} \mathrm{~mol} / \mathrm{dm}^{3}$. For BRF II. curves are shown for the three interference orders $m=1,2$, and 3 . The pulse durations increased with the interference order (spectral filter width narrows with interference order. see fig. 7 ). The pulse durations obtained with BRF I ( $m=2, \phi$ around $45^{\circ}$ ) are between the $m=2$ and $m=3$ results of BRF II $\left(\phi(m=2)\right.$ around $26^{\circ}$ and $\phi(m=3)$ around $32^{\circ}$ ). These pulse duration dependences on $m, \phi$ and $\psi$ are in good agreement with fig. 6 .

It should be noted that in fig. 12 the same absorber concentration was applied as for the curves 2 in fig. 11. But this time shorter pulse durations were obtained (e.g. for the laser without birefringent filter $\Delta t_{\mathrm{L}}=63 \mathrm{fs}$ in fig. 12 versus $\Delta t_{\mathrm{L}} \approx 97 \mathrm{fs}$ in fig. 11 ). For taking the data of fig. 12 the laser was newly aligned. 


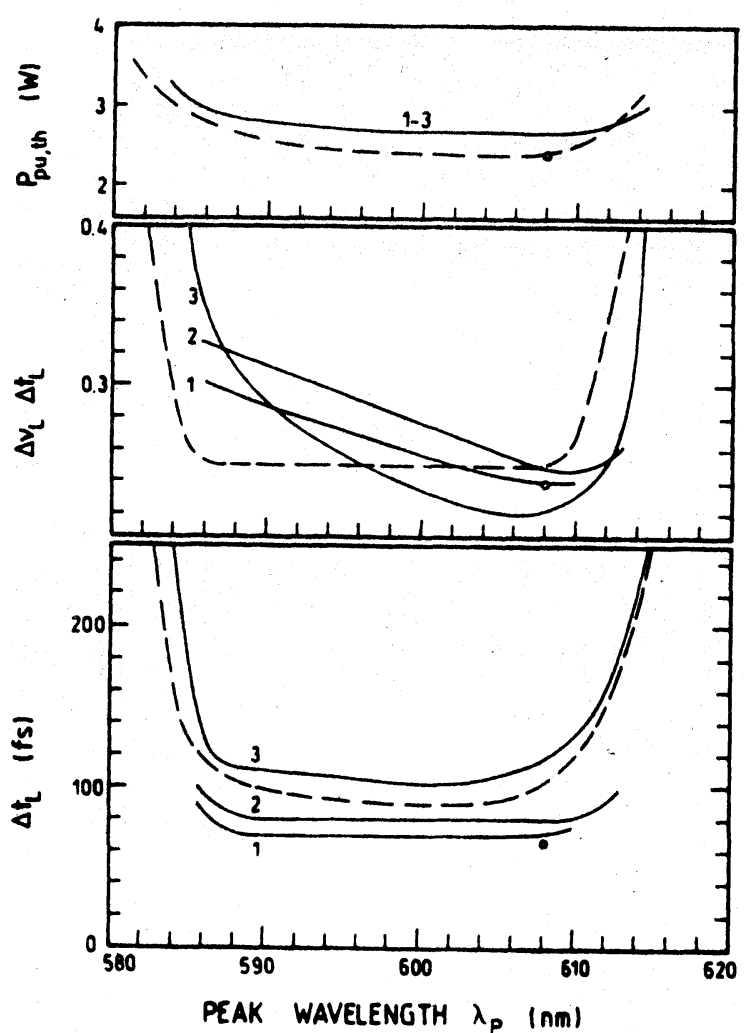

Fig. 12. Frequency tuning with BRF II (solid curves) at different interference orders ( $1: m=1,2: m=2.3: m=3)$ and with BRF I (dashed curve). Saturable absorber is TCVEBI of concentration $C_{a}=9 \times 10^{-5} \mathrm{~mol} / \mathrm{dm}^{3}$. Circles indicate results without birefringent filter.

The absorber jet seems to be nearer to the focal region of the absorber cavity resulting in a stronger selfphase modulation. A stronger self-phase modulation in the prism pair balanced laser operation (positive frequency chirp is compensated by negative group velocity dispersion of prism-pair; soliton-like pulse formation [12.30,34]) shows up in shorter durations and smaller bandwidth products $\Delta \nu_{\mathrm{L}} \Delta t_{\mathrm{L}}$ [33.34]. In the prism-pair balanced soliton-like pulse formation regime the steady-state pulse duration is determined mainly by the amount of self-phase modulation and it is limited by the effective amplification width determined by the gain and loss medium and the mirror reflectivities [12,33,34.37]. The saturable absorber is needed in the transient femtosecond pulse formation process.

\subsection{Spatial filter tuning}

For aperture tuning $[10.13,38]$ a $2 \mathrm{~mm}$ wide slit $A$, was positioned between prism PI and the end mirror M1 of the resonator (see fig. 1), and the slit A was transversely tuned. In fig. 10 the aperture tuning (dashed curves) is compared with the birefringent filter tuning (solid curves). The same absorber (DQOCI) of equal concentration $\left(C_{\mathrm{a}}=7.4 \times 10^{-5}\right.$ $\mathrm{mol} / \mathrm{dm}^{3}$ ) is used in both cases. The fine tuning of the slit allows the generation of pulses of duration $\Delta t_{L}<100$ fs only in a small spectral region between $590.5 \mathrm{~nm}$ and $592 \mathrm{~nm}$. There was no spectral modulation observed in the spatial filter tuning. The spatial spectrum dispersion $\partial \tilde{\nu} / \partial x$ at the spatial filter position is $\partial \tilde{\nu} / \partial x=830 \mathrm{~cm}^{-1} / \mathrm{mm}$ for our experimental situation (prism separation $35 \mathrm{~cm}$. fused silica Brewster prisms). For a spectral pulse width of $\Delta \tilde{\nu} \simeq 250 \mathrm{~cm}^{-1}$ the spatial spreading is $\Delta x=0.3 \mathrm{~mm}$ which is approximately half of the laser beam diameter (fwhm).

\section{Conclusions}

In this paper the frequency tuning of a cw pumped linear passive mode-locked prism-pair balanced rhodamine $6 \mathrm{G}$ dye laser was studied by azimuthal angle adjustment of single birefringent filter plates. Pulse durations below 100 fs were achieved over a wide tuning range (from 585 to $620 \mathrm{~nm}$ ). The tuning range of the applied rhodamine $6 \mathrm{G}$ femtosecond dye laser might be extended beyond $620 \mathrm{~nm}$ out to about 630 $\mathrm{nm}$ by using laser mirrors with high reflectivity in this wavelength region and increasing the reflectivity of the output mirror to about $99 \%$ in this spectral region [11]. Other saturable absorbers like DASBTI $[3,39,40]$, pinacyanol chloride $[41]$ or TCETI tetrafluoroborate [11] may be applied for the rhodamine $6 \mathrm{G}$ dye laser and similar tuning results are expected.

The described technique may be applied as well to group velocity dispersion balanced ring CPM dye lasers as to antiresonant ring CPM dye lasers [1]. Using other amplifying dye-saturable dye combinations frequency tunable sub- 100 fs pulse may be gen- 
erated in other spectral regions between about 490 $\mathrm{nm}$ and about $900 \mathrm{~nm}$ as well.

\section{Acknowledgements}

The authors are indepted to $M$. Schäffner for the fluorescence quantum yield measurements of $\mathrm{DQOCI}$ and TCVEBI, and to Dr. V. Petrov for fruitful discussions. They thank the Deutsche Forschungsgemeinschaft for financial support and the Rechenzentrum of the University for allocation of computer time.

\section{References}

[1] J.C. Diels, in: Dye laser principles with applications, eds. F.J. Duarte and L.W. Hillman (Academic Press. Boston. 1990) pp.41. *

[2] A. Penzkofer. Appl. Phys. B 46 (1988) 43.

(3) P.M.W. French. J.A.R. Williams and J.R. Taylor, Revue Phys. Appl. 22 (1987) 1651.

[4] B.V. Bondarev, V.I. Prokhorenko. G.D. Rodionov, V.B. Sorokin. Yu.L. Slominskii and E.A. Tikhonov. Sov. J. Quantum Electron. 19 (1989) 20.

[5] N. Michailov. T. Deligeorgiev, V. Petrov and I. Tomov, Optics Comm. 70 (1989) 137.

[6] N.I. Michailov, I.P. Christov and I.V. Tomov. Appl. Phys. B 51 (1990) 171 .

[7] N.H. Rizvi. M.M. Opalinska. P.M.W. French and J.R. Taylor. Optics Comm. 80 (1990) 57.

[8] P.K. Benicewicz. J.P. Roberts and A.J. Taylor. Optics Letters $16(1991) 925$.

[9] J.-C. Diels. J.J. Fontaine. I.C. McMichael. B. Wilhelmi, W. Dietel and D. Kühlke. Sov. J. Quantum Electron. 13 (1983) 1562.

[10] P.M.W. French and J.R. Taylor, Optics Lett. 13 (1988) 470.

(11) N.I. Michailov, T.G. Deligeorgiev, I.P. Christov and I.V. Tomov, Opt. Quantum Electron. 22 (1990) 293.

[12] W. Bäumler and A. Penzkofer, Opt. Quantum Electron., 24 (1992) 313.

(13) J. Dobler. H.H. Schulz and W. Zinth. Optics Comm. 57 (1986) 407.

[14] A. Penzkofer and W. Bäumler. Opt. Quantum Electron. 23 $(1991)+39$.

[15] M.D. Dawson, T.F. Boggess, D.W. Garvey and A.L. Smirl. Optics Comm. 60 (1986) 79.
[16] Z.A. Yasa and N.N. Amer. Optics Comm. 36 (1981) 406

[17] A. Penzkofer and W. Bäumler, Opt. Quantum Electron. 23 (1991) 727.

[18] W. Bäumler and A. Penzkofer, Chem. Physs. 140 (1990) 75.

[19] W. Bäumler and A. Penzkofer. Chem. Phys. 142 (1990) 431.

[20] A. Penzkofer and W. Leupacher. J. Luminesc. 37 (1987) 61.

[21] V. Sundström and T. Gillbro. Appl. Phỳs. B 31 ( 1983 ) 235.

[22] D.R. Preuss and J.L. Gole, Appl. Optics 19 (1980) 702.

[23] 1.J. Hodgkinson and J.I. Vukusic. Appl. Optics 17 (1978) 1944.

[24] H. Schillinger and A. Penzkofer, Optics Comm. 68 (1988) 45.

[25] W. Demtröder, Laser spectroscopy basic concepts and instrumentation, Springer Series in Chemical Physics. Vol. 5 (Springer. Berlin. 1981 ) pp. 389.

[26] G. Li and Y. Li, Appl. Optics 29 (1990) 3462.

[27] D.E. Spence, P.N. Kean, and W. Sibbett. Optics Lett. 16 (1991) 42.

[28] U. Keller. G.W. 'thooft, W.H. Knox and J.E. Cunningham. Optics Lett. 16 (1991) 1022.

[29] L. Spinelli. B. Couilland. N. Goldblatt. C. Seaton and D.K. Negus, Proc. VIIth International Symposium on Ultrafast processes in spectroscopy, Bayreuth 1991. ed. A. Laubereau (IOP Publishing, Bristol), to be published.

[30] J.A. Valdmanis and R.L. Fork. IEEE J. Quant. Electron. QE$22(1986) 112$.

[31] O.E. Maninez, R.L. Fork and J.P. Gordon. Optics Lett. 9 (1984) 156.

[32] W.L. Nighan. T. Gong and P.M. Fauchet. IEEE J. Quant. Electron. QE-25 (1989) 2476.

[33] A. Penzkofer. M. Wittmann. W. Bäumler and V. Petrov. Appl. Optics, to be published.

[34] A. Finch. G. Chen, W. Sleat and W. Sibbett. J. Mod. Opt. 35 (1988) 345.

[35] P. Sperber and A. Penzkofer, Opt. Quant. Electron. 18 (1986) 145

[36] F. Salin and A. Brun, J. Appl. Phys. 61 (1987) 4736

[37] A. Penzkofer and W. Bäumler, in: Lasers 91 . ed. F.J. Duante (STS Press. McLean, VA, 1992), to be published.

[38] H. Kubota. K. Kurokawa and M. Nakazawa. Optics Lett. $13(1988) 749$

[39] C.V. Shank, in: Ultrashort laser pulses. ed. W. Kaiser, Topics in Appl. Phys. Vol. 60 (Springer. Heidelberg. 1988) Ch. 2. pp. 5.

[40] P.M.W. French, M.D. Dawson and J.R. Taylor, Optics Comm. 56 (1986) 430.

[41] Ph. Hébern. T. Gustavsson and J.C. Mialocq, Laser '91 Laserion. Laser in Science. International Laser Symposium. München (Germany). Cochairs D. Basting and M. Stuke.

[42] American Institute of Physics Handbook. 3rd Edition. ed. D.E. Gray (McGraw Hill. New York, 1972) p. 6-27. 